\title{
Telomere repeat amplification protocol (TRAP) in situ reveals telomerase activity in three cell types in effusions: malignant cells, proliferative mesothelial cells, and lymphocytes
}

\author{
Nooreldin Zendehrokh ${ }^{1}$ and Annika Dejmek ${ }^{2}$ \\ ${ }^{1}$ Department of Clinical Pathology and Cytology and ${ }^{2}$ Department of Laboratory Medicine, Malmö University \\ Hospital, Lund University, Malmö, Sweden
}

\begin{abstract}
Telomerase Repeat Amplification Protocol (TRAP) in situ was performed on cytospin preparations from 65 effusions from the serous cavities (45 pleural and 19 ascitic fluids and one pericardial fluid) submitted for routine diagnosis and the results were correlated to cytological morphology. Three types of cells with nuclear fluorescence were identified: malignant cells, hyperplastic mesothelial cell and lymphocytes. Of 38 cytologically malignant effusions, 12 showed strong reactivity in all malignant cells, three strong reactivity in part of the malignant population, whereas 12 showed moderate reactivity in the whole and five in part of the malignant population, respectively. In five malignant effusions weak reactivity was found in all (one case) and in scattered (four cases) malignant cells. Two effusions contained telomerase-negative malignant cells. Two pleural and two ascitic fluids contained proliferative mesothelial cells with weak or, in one case, moderate reactivity. Lymphocytes usually showed weak telomerase activity. Telomerase was expressed in almost all malignant tumours metastatic to serous cavities. Heterogeneity in tumour populations was demonstrated, which may have diagnostic implications, especially in cytology. Weak or moderate reactivity was found in lymphocytes and in some mesothelial proliferations and may explain the low specificity for malignancy sometimes obtained with the TRAP extract method. The weak reactivity found in lymphocytes may reduce the specificity when the extract method is used but causes no diagnostic problem with the TRAP in situ method. Modern Pathology (2005) 18, 189-196, advance online publication, 24 September 2004; doi:10.1038/modpathol.3800278
\end{abstract}

Keywords: telomerase; effusion; cytology; cancer; mesothelial cells; lymphocytes; TRAP in situ

The telomeres are essential for maintaining the integrity and stability of linear eukaryotic genomes. They are special structures found at the ends of the chromosomes and consist of tandem repeat DNA sequences and associated proteins. The addition of telomeric DNA to the telomeres is mainly achieved by telomerase, a ribonucleoprotein complex. Telomerase is not active in quiescent somatic cells but is usually required for cell immortalization and plays an important role in oncogenesis. ${ }^{1-7}$ Using the telomere repeat amplification protocol (TRAP), a PCR-based assay described by Kim et $a l^{8}$ in 1994

Correspondence: Dr A Dejmek, MD, PhD, Department of Laboratory Medicine, Division of Pathology, Malmö University Hospital, S-205 02 Malmö, Sweden.

E-mail: annika.dejmek@pat.mas.lu.se

Received 29 March 2004; revised and accepted 12 July 2004; published online 24 September 2004 telomerase activity has been demonstrated in over $80 \%$ of malignant tumors from various organs. ${ }^{9}$ Thus, telomerase activity might play a role as a diagnostic marker of malignancy.

Effusions represent a major diagnostic challenge. The cellular content is often complex and the identification of malignant cells may be difficult especially if they are scanty. Further, the distinguishing of mesothelioma or adenocarcinoma cells from benign hyperplastic mesothelial cells is a wellrecognized difficulty.

When applied to effusions the TRAP assay has shown considerable variation in sensitivity and specificity ${ }^{10-17}$ and the value of the assay as an adjunct in the diagnosis of malignancy is controversial.

Telomerase activity is usually compared with cytological diagnosis. Braunschweig et $a l^{17}$ find a lower sensitivity for TRAP assay than for cytological 
diagnosis. Toshima et $a l^{13}$ report similar figures. In their review, Hess and Highsmith ${ }^{11}$ report a higher sensitivity for telomerase than for conventional cytology. Since the assay is performed on cell extracts it allows no morphological correlation that might elucidate these differences.

Therefore, the TRAP method was further developed by Ohyashiki et $a l^{18}$ to an in situ method performed on cytospin preparations and suitable especially for cytological material. It has been tested on urine, effusions, bronchial brushings, etc and has shown a high sensitivity and specificity for malignancy. ${ }^{19-22}$

The method has now been applied to a larger series of unselected effusions to analyze in detail the reasons for false-positive and -negative results obtained with the extract method and evaluate the potential of TRAP in situ as a diagnostic marker of malignancy. A detailed correlation has been made between telomerase reactivity and cellular content and morphology, including the results of the ancillary techniques used in the laboratory when applied. Further, a semiquantitative analysis of the telomerase reactivity has been made.

\section{Materials and methods}

The study comprises 65 effusions submitted fresh to the Department of Pathology and Cytology, Malmö University Hospital from March 2002 to January 2004. All effusions allotted for diagnosis to one of the authors (AD) where the amount of fluid was sufficient and where follow-up is available, are included.

For preparation of routine stained smears $20 \mathrm{ml}$ of fluid were spun at $3000 \mathrm{rpm}$ for $10 \mathrm{~min}$ in a HettichRotica centrifuge, the supernatant was removed, and the cell pellet was smeared onto slides. Two air-dried Giemsa-stained slides and two wet-fixed Htx-eosin-stained slides were made from each specimen.

Effusions containing atypical or malignant epithelial or mesothelial cells or epithelial-like cells that could not be characterized further morphologically were also subjected to immunocytochemical stains according to the routines of the department, based on the results of previous research (a small panel consisting of carcinoembryonic antigen (CEA), BerEp4 and epithelial membrane antigen (EMA) where predominant membrane staining was evaluated as a separate parameter or an extended panel also including HBME-1, thrombomodulin, vimentin, Ca125, cytokeratin 7 (CK7), cytokeratin 20 (CK 20), and cytokeratin 5 (CK 5) and Sialyl-Tn) and analysis of the hyaluronan content with a previously described high-performance liquid chromatography (HPLC)-based method in accordance with the diagnostic routines of the department. ${ }^{23-25}$ The 'true' diagnoses were based on all available information in every single case; cytological, histological and autopsy diagnoses, clinical information, including the results of imaging techniques and clinical follow-up. Cases without a final diagnosis were not included.

The in situ TRAP method has previously been described in detail and was applied with slight modifications. ${ }^{18,22}$

A volume of $10 \mathrm{ml}$ of the fresh fluid were spun (1500 rpm, $5 \mathrm{~min}$ ), the pellet was resuspended in hypotonic solution (10 mM Tris, $10 \mathrm{mM} \mathrm{KCl,} 1 \mathrm{mM}$ $\mathrm{MgCl}_{2}$ ), and carefully mixed, and hypertonic solution $\left(274 \mathrm{mM} \mathrm{NaCl}, 5.4 \mathrm{mM} \mathrm{KCl}, 16.2 \mathrm{mM} \mathrm{Na}_{2} \mathrm{HPO}_{4}\right.$, $2.9 \mathrm{mM} \mathrm{KH}_{2} \mathrm{PO}_{4}$ ) was added. The cell suspension was cytospun (400 rpm, $3 \mathrm{~min}$ ) onto nonfluorescent slides and dried with cold air. A measure of $25 \mathrm{ml}$ of a solution containing $20 \mathrm{nM}$ Tris-HCl $(\mathrm{pH} 8.3)$, $1.5 \mathrm{mM} \mathrm{MgCl}_{2}, 63 \mathrm{mM} \mathrm{KCl}, 0.05 \%$ Tween-20, $1 \mathrm{mM}$ EGTA, that is, ethylene glycol-bis (beta-aminoethyl eter)- $N, N, N, N$-tetraacetic acid, $50 \mathrm{mM}$ dNTPs, $1 \mu \mathrm{g}$ of $\mathrm{T} 4$ gene 32 protein, bovine srum albumin $(0.1 \mathrm{mg} /$ $\mathrm{ml}), 2 \mathrm{U}$ of Taq DNA polymerase, and $10 \mathrm{pM}$ FITClabeled TS forward-primer (5'-AAT CCG TCG AGC AGA GTT-3') was added to a chamber frame (65 $\mu$ l) that had been placed over each specimen to hold the reaction solutions, and the slides were incubated for $30 \mathrm{~min}$ at $22^{\circ} \mathrm{C}$. After TS extension, $25 \mu \mathrm{l}$ of the same solution but with 10 pmol of FITC-labeled CX reverse-primer (5'-CCC TTA CCC TTA CCC TTA CCC TTA-3 ${ }^{\prime}$ ) instead of TS forward primer were added. The reaction chamber was sealed with a coverslip and heated to $90^{\circ} \mathrm{C}$ for $1.5 \mathrm{~min}$ to inactivate telomerase. The telomerase reaction product was then amplified using a PTC-100 ${ }^{\mathrm{TM}}$ Programmable Thermal Controller MJ Research, INC. (SDS Scandinavian Diagnostic).

The PCR conditions were 30 cycles at $94^{\circ} \mathrm{C}$ for $30 \mathrm{~s}, 50^{\circ} \mathrm{C}$ for $30 \mathrm{~s}$, and $72^{\circ} \mathrm{C}$ for $90 \mathrm{~s}$. The chamber frames and top liners were removed completely, and the slides were rinsed in room-tempered PBS buffer and then sealed with a coverglass using $\mathrm{DAKO}^{\circledR}$ Fluorescent mounting medium.

For each specimen slides without Taq polymerase, without primer, and with all reagents but not subjected to PCR were included. Further, one cytospin slide from each specimen was Giemsa stained.

The routine stained smears and the Giemsastained cytospins were examined thoroughly and the cell types found in each case were noted and their proportions estimated. A semiquantitative analysis of the overall cellularity in the routine stained smears (scanty, moderate, abundant) and the various cell types (mesothelial cells, macrophages, lymphocytes, granulocytes, atypical and malignant cells or cells suspicious for malignancy) was made and the results were correlated to the outcome of the TRAP in situ assay. The TRAP in situ slides were examined with a microscope (Olympus BX51) allowing alternation between fluorescence (with a B-filter) and phase contrast (U Plane Fluorite $\times 40$ Phase Contrast objective) to enable the identification 
also of telomerase-negative cells. Based on nuclear and cytoplasmic morphology and cellular arrangement an attempt to identify all cell types found in the routine stained smears was made with phasecontrast microscopy. Then the type and intensity of fluorescence in the identified celltypes was evaluated with the microscope in the fluorescent mode. Nuclear fluorescence only or nuclear fluorescence stronger than cytoplasmic fluorescence was considered positive. The intensity was assigned to three groups, weak $(+)$, moderate $(++)$, and strong $(+++)$ and an attempt was made to estimate whether all or only part of the cell populations identified showed reactivity.

All evaluations were made by both authors independently, to avoid bias, in a blinded fashion without knowledge of the morphologically based diagnoses.

Since Malmö University Hospital is the town's only referral hospital a search of the laboratory files assured that no histologically verified diagnosis was missed.

\section{Results}

The detailed results for the various cell types are given in Table 1. In all cases, the same cell types could be identified both in the routine stained smears and in the TRAP in situ preparations.

\section{Cytologically Malignant Effusions}

The reactivity in malignant cells according to tumour type are given in Table 2. Telomerase reactivity was found in 36 of 38 effusions (29 pleural, eight peritoneal, one pericardial) containing well-preserved carcinoma cells. In 12 of the cases strong nuclear fluorescence was seen in all malignant cells (Figures 1 and 2), in three cases strong reactivity was found in part of the malignant population, whereas 11 cases showed moderate reactivity in the whole, and five in part of the malignant population, respectively. In one malignant effusion weak reactivity was found in all

Table 1 Telomerase reactivity according to cell type

\begin{tabular}{lcccc}
\hline Telomerase reactivity & Malignant cells & $\begin{array}{c}\text { Nonproliferative } \\
\text { mesothelial cells }\end{array}$ & $\begin{array}{c}\text { Proliferative } \\
\text { mesothelial cells }\end{array}$ & Lymphocytes \\
\hline++ & 14 & 0 & 0 & 0 \\
++ & 16 & 0 & 1 & 0 \\
+ & 6 & 0 & 4 & 5 \\
Total telomerase pos cases & 36 & 0 & 6 & All cases where lymphocytes \\
Telomere identified &
\end{tabular}

Table 2 Characterization of telomerase activity in malignant cells according to tumour type and primary

\begin{tabular}{|c|c|c|c|c|c|c|c|c|c|c|}
\hline \multirow[t]{3}{*}{ Tumour type } & \multirow[t]{3}{*}{ Primary } & \multirow[t]{3}{*}{ No. of cases } & \multicolumn{7}{|c|}{ TRAP in situ } & \multirow[t]{3}{*}{ Any positivity } \\
\hline & & & \multirow[t]{2}{*}{$\mathrm{Neg}$} & \multicolumn{3}{|c|}{ All } & \multicolumn{3}{|c|}{ Part } & \\
\hline & & & & + & ++ & +++ & + & ++ & +++ & \\
\hline \multirow[t]{8}{*}{ Adenocarcinoma } & Lung & 10 & & 1 & 1 & 5 & 2 & & 1 & 10 \\
\hline & Breast & 6 & & & 2 & 2 & & 2 & & 6 \\
\hline & Pancreas & 2 & & & 2 & & & & & 2 \\
\hline & Ovary & 6 & & & 1 & 3 & & 1 & 1 & 6 \\
\hline & Cervix uteri & 1 & & & 1 & & & & & 1 \\
\hline & Colon & 1 & & & & & 1 & & & 1 \\
\hline & Unknown & 3 & 1 & & 1 & & & 1 & & 2 \\
\hline & Fallopian tube & 1 & & & 1 & & & & & 1 \\
\hline Squamous carcinoma & Lung & 2 & & & & & 1 & & 1 & 2 \\
\hline Non-small cell carcinoma & Lung & 1 & & & 1 & & & & & 1 \\
\hline Uroepithelial carcinoma & Bladder & 1 & & & & 1 & & & & 1 \\
\hline Renal carcinoma & Kidney & 1 & 1 & & & & & & & 0 \\
\hline Malignant fibrous histiocytoma & Soft tissue (leg) & 1 & & & 1 & & & & & 1 \\
\hline Mesothelioma & & 2 & & & & 1 & & 1 & & 2 \\
\hline Total & & 38 & 2 & 1 & 11 & 12 & 4 & 5 & 3 & 36 \\
\hline
\end{tabular}


tumour cells and in four cases in scattered malignant cells. There were two mesotheliomas among the cases. One mesothelioma showed strong reactivity in the whole population, whereas moderate reactivity in some of the cells was found in the other case. Two effusions contained poorly differentiated malignant cells without telomerase reactivity. One case was an ascitic fluid from a patient with an adenocarcinoma originating in the Fallopian tube. The other case was a pleural effusions with a small amount of cells highly suspicious for malignancy, probably representing a kidney carcinoma (autopsy verified). As for the telomerase-positive cases there was no trend towards correlation between telomerase reactivity and primary tumour site and differentiation grade.

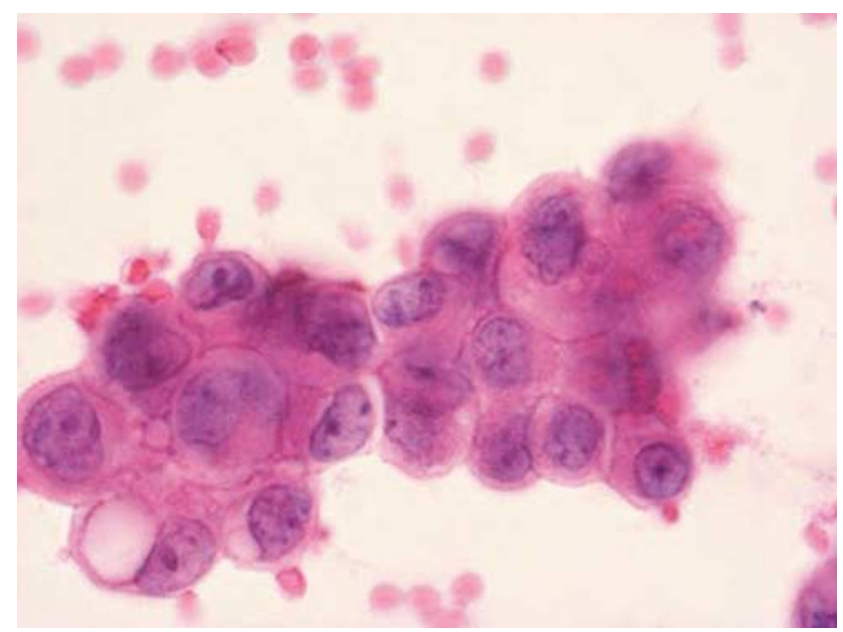

Figure 1 Pleural effusion containing metastatic adenocarcinoma cells from a previously diagnosed lung carcinoma. A cluster of moderately atypical malignant cells with partly overlapping nuclei in routine preparation $(\mathrm{HE}, \times 320)$.

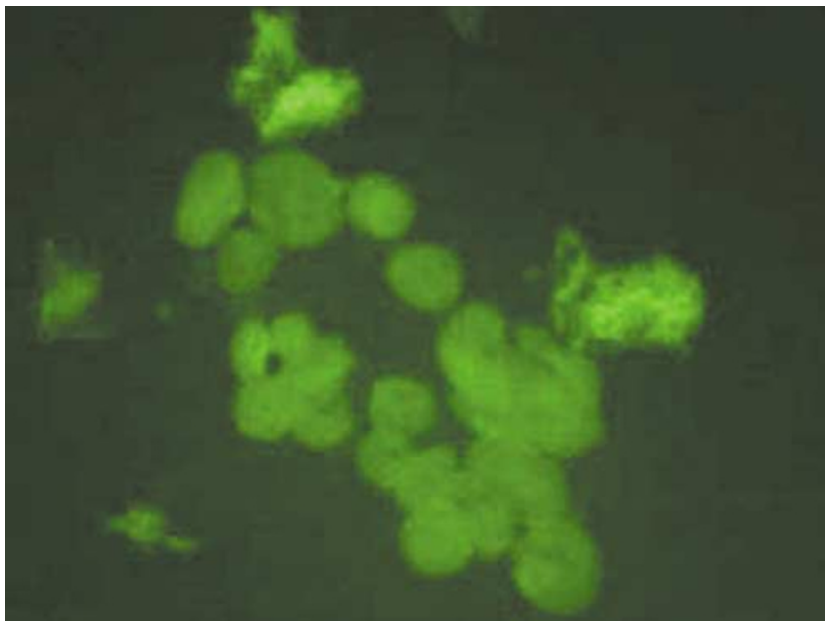

Figure 2 A cluster of similar malignant cells (same case as Figure 1) with strong nuclear fluorescence (TRAP in situ, $\times 320$ ).

\section{Cytologically Benign Effusions}

In all, 16 effusions (10 pleural, six peritoneal fluids) were cytologically characterized as benign or 'reactive' with unspecific features. They contained a mixture of macrophages, lymphocytes, neutrophils, sometimes eosinophils and a small or moderate amount of mesothelial cells without cellular or architectonic atypia. Lymphocytes usually showed weak telomerase activity and cytoplasmic fluorescence was seen in neutrophils and especially in eosinophils,. There was no reactivity in mesothelial cells in these cases.

\section{Effusions with Proliferative Mesothelial Cells}

In all, 11 effusions (seven pleural, four peritoneal fluids), contained proliferative mesothelial cells. In five of these, the mesothelial cells showed nuclear atypia and a tendency to form dense, sometimes papillary clusters but the effusions were finally evaluated as probably benign.

In six cases, no reactivity was seen in mesothelial cells. Weak reactivity in mesothelial cells was found in two ascitic fluids, both from patients with chronic hepatitis and in two pleural effusions, both from patients with severe cardiac failure. Moderate reactivity in mesothelial cells was seen in one specimen. This was a peritoneal fluid from a patient with liver cirrhosis and septicaemia. The patient died shortly afterwards, no autopsy was performed and thus, no definite diagnosis obtained. Since the telomerase activity was found in cells with undoubtable mesothelial morphology the case was not excluded (Figures 3 and 4). As for the rest of the cases further investigations and clinical follow-up revealed no malignancy.

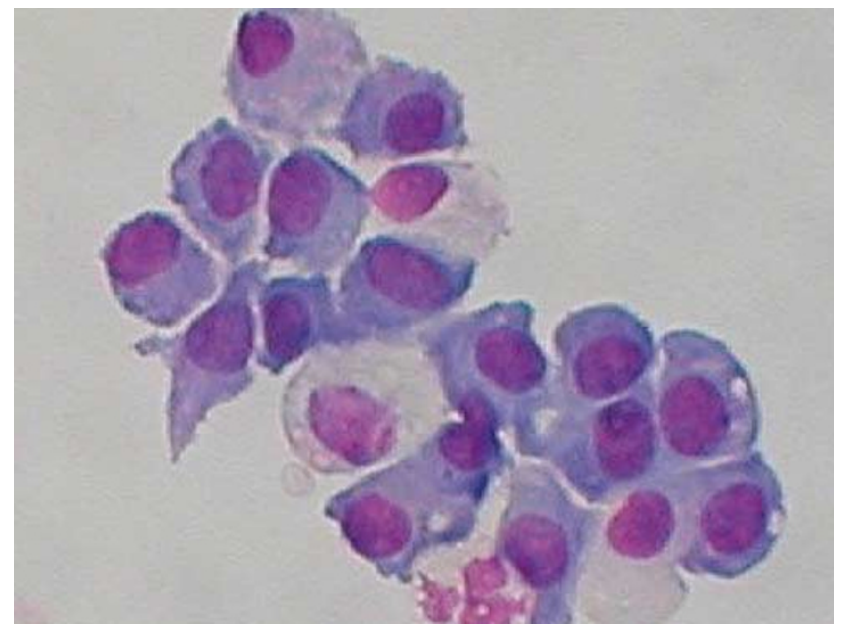

Figure 3 Ascitic fluid containing inflammatory cells, macrophages and mesothelial cells from a patient with liver cirrhosis. A small two-dimensional sheet of mesothelial cells in routine preparation (Giemsa, $\times 320$ ). 


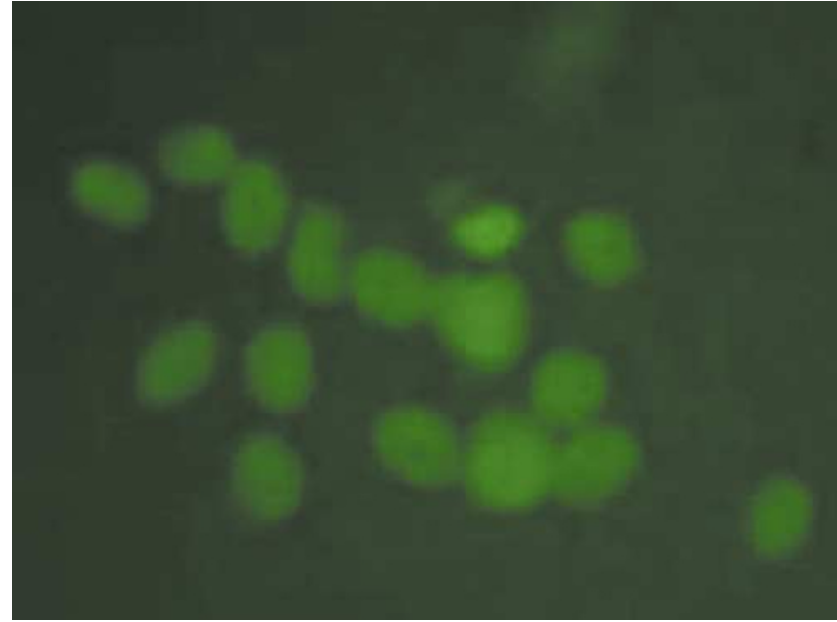

Figure 4 A similar group of mesothelial cells (same case as Figure 3) with moderate nuclear fluorescence (TRAP in situ, $\times 320$ ).

\section{Discussion}

\section{Correlation between Cell Type and Telomerase Activity in Effusions}

Telomerase activity has been demonstrated in a majority of human malignant tumours. However, when the TRAP assay has been applied to effusions the sensitivity and specificity for malignancy has varied. In a previous TRAP in situ study, ${ }^{18}$ we demonstrated telomerase activity in 7/9 malignant effusions, a result that corresponds well to the results generally obtained with the extract method when histological tumour material is used. The activity was found in morphologically malignant cells.

Our present results based on an extended material indicate that three cell types in effusions may present nuclear telomerase activity: morphologically malignant cells, lymphocytes, and proliferating mesothelial cells. Neutrophils and especially eosinophils, and macrophagi showed cytoplasmic but never nuclear fluorescence.

These findings may explain, to some extent, the reported variation in specificity of the TRAP extract method. Thus, the presence of lymphocytes in the effusion may explain some false-positive results obtained with the TRAP extract method. ${ }^{15}$ Reactivity in lymphocytes is well known ${ }^{26,27}$ and has previously been suggested as one explanation for low specificity for malignancy in effusions. This assumption is supported by our finding of weak reactivity in lymphocytes in many of the specimens. In their series of 144 effusions Yang et $a l^{14}$ found telomerase activity in three benign fluids, all coming from patients with tuberculosis. This is an interesting observation since tuberculosis is known to cause lymphocytic effusions.

The telomerase activity in lymphocytes does not hamper the diagnosis when the TRAP in situ method is used (unless lymphoma is a differential diagnosis) since the cells can be identified morphologically.

\section{Sensitivity for Malignancy of TRAP In Situ}

Overall, 36/38 malignant effusions contained telomerase-positive malignant cells (sensitivity 95\%, confidence interval 82-99\%). One of the telomerasenegative cases was a kidney carcinoma. According to the literature, kidney carcinomas may lack telomerase activity. Thus, it can be demonstrated with TRAP in situ, that telomerase is, with few exceptions, expressed in all malignant tumours growing in the serous cavities.

Tumour tissues are composed of several different cell types and contain inhibitory substances that can greatly affect the accuracy of any assay. The source and the quality of the sample influence the result when telomerase is extracted from tissues or cells. ${ }^{28}$ Both benign and malignant clinical cytological samples are often hemorrhagic, which leads to effects of interfering substances and inhibition (eg, erythrocytes, hemoglobin) of PCR. With the TRAP extract method this has been shown for fine-needle aspirates and for effusions. ${ }^{10,12,29}$ The varying results for effusions when the TRAP extract method is used may be due to extraction problems. CHAPS lysis buffer is part of the TRAPeze detection kit, but other extraction detergents exist, which may be better suitable for effusions. Also, during the extraction procedure, especially from tumor specimens, PCR inhibitors are diluted and may be removed by additional re-extraction steps. ${ }^{30}$ Preliminary results of direct comparisons from our laboratory indicate that the in situ method is more sensitive than the extract method and that the sensitivity for the extract method may be improved by additional extraction steps (data not shown).

Some studies reporting low sensitivity for malignancy also report poor sample preservation and poor RNA integrity. ${ }^{17}$

Further, in the environment of large fluid volumes, the detection of telomerase activity in a small percentage of cells is difficult.

With the in situ method, the intact cells are spun onto slides and immediately fixed and therefore telomerase activity may be better preserved and the cells may be protected from inhibitory substances in the fluid or released from other cell types during the extraction process.

Some of our malignant fluids were heavily blood stained, but this did not seem to effect telomerase activity when the in situ method is used.

We noted that telomerase reactivity was not equally strong in all cell types. Only malignant cells showed strong nuclear fluorescence. Thus, based on our data so far, strong nuclear telomerase activity in effusions is a high probability marker for malignancy. It is of particular utility when the malignant 
cells are scanty and easily could be overlooked in conventional stains, where non-neoplastic cell types may dominate. This is a well-known problem in effusion cytology. In our opinion, even a few cells with strong nuclear fluorescence indicate malignancy. The quantification of the staining intensity was entirely subjective, but since the scores independently assigned by the two authors differed at most less than one step, we believe that the observed differences reflect true conditions. Thus, a further possibility to increase the diagnostic utility of the in situ method might be the absolute quantification of the staining intensity. Attempts to develop further the TRAP in situ method to allow objective quantification of the reactivity intensity have therefore been started in our laboratory.

In approximately one-third of the telomerasepositive tumours only part of the malignant population expressed telomerase activity. The intensity of the fluorescence signal was weaker in the cases where only part of the cell population was positive $\left(\chi^{2}, P<0.05\right)$. It cannot be concluded if this reflects a true heterogeneity in the upregulation of telomerase or a loss of telomerase activity in late tumour stage, perhaps when the cells are shed into the effusion. This finding may have diagnostic implications, especially in effusion cytology, since some malignant effusions only contain a small number of malignant cells.

\section{Telomerase Reactivity in Mesothelial Cells}

Both benign and malignant effusions usually contain varying amounts of mesothelial cells. When the TRAP extract method shows telomerase activity in effusions without cytological signs of malignancy, it may be due to the presence of malignant cells that could not be identified, or the reactivity may come from mesothelial cells or lymphocytes in the specimen. With the TRAP in situ method, we could demonstrate that non-neoplastic mesothelial cells may express weak or moderate telomerase activity.

In cases where it is impossible to differentiate between mesothelial cells and malignant metastatic or mesothelioma cells it is obviously a drawback that telomerase activity is also found in reactive mesothelial cells. This is a problem especially when a mesothelioma might be considered since the means to differentiate between mesothelioma and hyperplastic mesothelial cells are limited. It is well known that atypical mesothelial cells may be difficult to differentiate from cancer cells and some previous studies indicate that telomerase can be expressed in proliferative mesothelial cells. Nagel et $a l^{31}$ demonstrated expression of the catalytic subunit of human telomerase in 6/15 benign effusions containing lymphocytes and mesothelial cells and also demonstrated weak telomerase activity in mesothelial cell lines with the TRAP extract method. In studies where telomerase activity has been tested for distinguishing between mesothelial proliferations and mesothelioma, reactivity has been demonstrated in mesothelial cell lines and in sections from reactive serosal lesions. ${ }^{32-34}$

In our series, three of the effusions showing reactivity in proliferative mesothelial cells were peritoneal fluids from patients with liver disease. Only two pleural effusions contained telomerasepositive mesothelial cells.

A number of underlying conditions are known to cause mesothelial proliferation, such as liver disease, uremia, and chronic heart failure. If telomerase reactivity in mesothelial cells can be correlated to special causes, the diagnostic consequences may be reduced.

With the in situ method, we could demonstrate that the reactivity in mesothelial cells was weak or, in one case only, moderate. As with all staining methods, the subjective quantification of intensity is difficult. It is reasonable to assume, however, that the weaker intensity observed in mesothelial proliferations represent a spurious telomerase reactivity in proliferative non-neoplastic cells compared with a higher telomerase activity in most malignant cells.

Strong reactivity was seen in one of the two mesotheliomas and was an alert to malignancy. However, the other mesothelioma showed moderate fluorescence intensity, indistinguishable from that seen in one of the non-neoplastic mesothelial proliferations (peritoneal fluid from a patient with severe liver disease). Thus, telomerase reactivity does not specifically discriminate between mesotheliosis and mesothelioma.

\section{Conclusions}

The TRAP in situ method allows analysis of the telomerase reactivity in effusions and offers possible explanations for the varying results obtained with the extract method. When telomerase activity can be correlated to cell type, it becomes clear that telomerase is expressed by most malignant tumors metastatic to the serous cavities (sensitivity for malignancy $95 \%$ ).

Thus, we assume that the lower sensitivity figures sometimes obtained with the extract method may be due to inhibition of telomerase and/or the lack of relevant cells in the preparation.

The heterogeneity in tumor cells observed with TRAP in situ may have diagnostic or prognostic implications, especially in effusion cytology since effusions may contain only small numbers of malignant cells.

Our results confirm that telomerase is not the ultimate marker of malignancy, the specificity in our material being $81 \%$, when all staining intensities are included. However, strong reactivity, also in single cells, is an indication of malignancy and therefore has a potential as an ancillary diagnostic tool in 
cases when the malignant cells are sparse. This is not uncommon in clinical cytological practice and is one of the occasions where adjuvant techniques may contribute to an increased diagnostic accuracy. Further development of the method to quantify the fluorescence signal might increase the clinical utility of TRAP in situ and such attempts are in progress in our laboratory.

The in situ method confirms telomerase reactivity in lymphocytes. The telomerase reactivity found in lymphocytes reduces the specificity for malignancy only when no correlation to morphology is possible and, thus, is no major problem with the in situ method.

Telomerase activity in proliferative mesothelial cells were in $3 / 5$ cases found in ascitic fluid from patients with liver disease. Liver disease is often associated with peritoneal fluid with florid mesothelial proliferations. Whether telomerase upregulation in mesothelial cells is correlated to specific disease entities remains to be elucidated. If this is indeed the case, the utility of TRAP in situ in diagnostic cytology might be further increased.

\section{Acknowledgements}

The study was supported by grants from the Swedish Cancer Fund (4737-B03-02XBB) and from the Malmö University Hospital Cancer Fund.

\section{Duality of interest}

No duality of interest.

\section{References}

1 Rhyu MS. Telomeres, telomerase and immortality. J Natl Cancer Inst 1995;87:884-894.

2 Blackburn EH. Structure and function of telomeres. Nature 1991;350:569-573.

3 Wright WE, Shay JW. Telomere dynamics in cancer progression and prevention; fundamental differences in human and mouse telomerase biology. Nat Med 2000;6:847-851.

4 Shay JW, Bacchetti S. A survey of telomerase activity in human cancer. Eur J Cancer 1997;33:787-791.

5 Shay JW, Wright WE. Telomerase activity in human cancer. Curr Opin Oncol 1996;8:66-71.

6 Cong YS, Wright WE, Shay JW. Human telomerase and its regulation. Microbiol Mol Biol Rev 2002;66: 407-425.

7 Granger MP, Wright WE, Shay JW. Telomerase in cancer and aging. Crit Rev Oncol Hematol 2002; 41:29-40.

8 Kim NW, Piatyszek MA, Prowse KR, et al. Specific association of human telomerase activity with immortal cells and cancer. Science 1994;266:2011-2015.

9 Piatyszek MA, Kim NW, Weinrich SL, et al. Detection of telomerase activity in human cells and tumors by a telomeric repeat amplification protocol (TRAP). Methods Cell Sci 1995;17:1-15.
$10 \mathrm{Mu}$ XC, Brien TP, Ross JS, et al. Telomerase activity in benign and malignant cytologic fluids. Cancer (Cancer Cytopathol) 1999;87:93-99.

11 Hess JL, Highsmith WE. Telomerase detection in body fluids. Clin Chem 2002;48:18-24.

12 Cunningham V, Markham N, Shroyer AL, et al. Detection of telomerase expression in fine-needle aspirations and fluids. Diagn Cytopathol 1998;18: 431-436.

13 Toshima S, Arai T, Yasuda Y, et al. Cytological diagnosis and telomerase activity of cells in effusions of body cavities. Oncol Rep 1999;6:199-203.

14 Yang CT, Lee MH, Lan RS, et al. Telomerase activity in pleural effusions: diagnostic significance. J Clin Oncol 1998;16:567-573.

15 Tangkijvanich P, Tresukosol D, Sampatanukul P, et al. Telomerase assay for differentiating between malignancy-related and nonmalignant ascites. Clin Cancer Res 1999;5:2470-2475.

16 Murakami J, Nagai N, Ohama K. Telomerase activity in body cavity fluid and peritoneal washings in uterine and ovarian cancer. J Int Med Res 1998;26:129-139.

17 Braunschweig R, Yan P, Guilleret I, et al. Detection of malignant effusions: comparison of telomerase assay and cytological examination. Diagn Cytopathol 2001; 24:174-180.

18 Ohyashiki K, Ohyashiki JH, Nishimaki J, et al. Cytological detection of telomerase activity using an in situ telomeric repeat amplification protocol assay. Cancer Res 1997;57:2100-2103.

19 Dejmek A, Yahata N, Ohyashiki K, et al. Correlation between morphology and telomerase activity in cells from exfoliative lung cytologic specimens. Cancer (Cancer Cytopathol) 2000;90:117-125.

20 Ohyashiki K, Yahata N, Ohyashiki JH, et al. A combination of semi-quantitative telomerase assay and in-cell telomerase activity measurement using exfoliated urothelial cells for the detection of urothelial neoplasia. Cancer 1998;83:2554-2560.

21 Yahata N, Ohyashiki K, Ohyashiki JH, et al. Telomerase activity in lung cancer cells obtained from bronchial washings. J Natl Cancer Inst 1998;90:684-690.

22 Dejmek A, Yahata N, Ohyashiki K, Ebihara Y, et al. In situ telomerase activity in pleural effusions: a promising marker for malignancy. Diagn Cytopathol 2000; 24:11-15.

23 Dejmek A, Hjerpe A. Reactivity of six antibodies in effusions of mesothelioma, adenocarcinoma, and mesotheliosis: stepwise logistic regression analysis. Cytopathology 2000;11:8-17.

24 Brockstedt U, Gulyas M, Dobra K, et al. An optimized battery of eight antibodies that can distinguish most cases of epithelial mesothelioma from adenocarcinoma. Am J Clin Pathol 2000;114:203-209.

25 Nurminen M, Dejmek A, Martensson G, et al. Clinical utility of liquid-chromatographic analysis of effusions for hyaluronate content. Clin Chem. 1994; 40:777-780.

26 Counter CM, Gupta J, Harley CB, et al. Telomerase activity in normal leukocytes and in hematologic malignancies. Blood 1995;85:2315-2320.

27 Norrback KF, Dahlenborg K, Carlsson R, et al. Telomerase activation in normal $B$ lymphocytes and non-Hodgkin's lymphomas. Blood 1996;88:222-229.

28 Saldanha SN, Andrews LG, Tollefsbol TO. Analysis of telomerase activity and detection of its catalytic subunit, hTERT. Anal Biochem 2003;315:1-21. 
29 Braunschweig R, Guilleret I, Delacretaz F, et al. Pitfalls in TRAP assay in routine detection of malignancy in effusions. Diagn Cytopathol 2001;25:225-230.

30 Gollahon LS, Holt SE. Alternative methods of extracting telomerase activity from human tumor samples. Cancer Lett 2000;159:141-149.

31 Nagel H, Schlott T, Schulz GM, et al. Gene expression analysis of the catalytic subunit of human telomerase (hEST2) in the differential diagnosis of serous effusions. Diagn Mol Pathol 2001;10:60-65.
32 Dhaene K, Hubner R, Kumar-Singh S, et al. Telomerase activity in human pleural mesothelioma. Thorax 1998;53:915-918.

33 Kumaki F, Kawai T, Churg A, et al. Expression of telomerase reverse transcriptase (TERT) in malignant mesotheliomas. Am J Surg Pathol 2002;26: $365-370$.

34 Foddis R, De Rienzo A, Broccoli D, et al. SV40 infection induces telomerase activity in human mesothelial cells. Oncogene 2002;21:1434-1442. 